of life, and then very resistant, we are warranted in considering this a genuine congenital deformity." Is it possible that this might also have been a fracture dislocation, though partially reduced, as the result of a violent manipulation at birth?

Concerning the scoliosis, it would seem that this, too, must be attributed to the injury. There is no roentgenographic evidence of the presence of any of the anomalous conditions mentioned by Bohm ${ }^{4}$ following developmental error occurring in embryonic life, nor is there any evidence of the wedge-shaped supernumerary vertebrae reported by Fitzwilliams ${ }^{5}$ and by Norbury. ${ }^{\circ}$ Apparently one must add injury at birth to the causes of so-called congenital scoliosis. ${ }^{i}$

\section{THE DELETERIOUS EFFECT OF THE ALKALIZATION OF INFANTS' FOOD *}

\section{ALFRED F. HESS, M.D. AND \\ LESTER J. UNGER, M.D. NEW YORK}

At the present time little regard is paid to the reaction of the milk preparations that are fed to babies. It is considered quite immaterial, for example, whether being not specifically mentioned. This is true of American, English, French and German textbooks. This neglect is all the more remarkable as for some years it has been appreciated that the salts play an important rôle in infant feeding; indeed, in every effort to construct an artificial food approximating mother's milk, the salt factor has been given particular consideration.

We wish to point out that it is by no means immaterial whether we render the baby's milk more or less alkaline: What effect an addition of alkali has on proteins and other caloric food factors it is impossible, in the present state of our knowledge, even to surmise ; but that this alteration has a decided effect on the vitamins is susceptible to proof. In a previous article we have shown ${ }^{\mathbf{1}}$ that orange juice, the prototype of antiscorbutics, and the one most relied on in infant feeding is essentially damaged within twenty-four hours by being rendered twentieth normal alkaline to phenolphthalein. In fact, orange juice of this faintly alkaline reaction was found to have lost its power to protect guinea-pigs against scurvy. It is evident, therefore, that the antiscorbutic vitamin is peculiarly sensitive to alkalization. We may add that this vitamin seems to be more sensitive to various physical and chemical influences than either the water-soluble or the fat-soluble vitamin. There is at present some difference of opinion as to the vulnerability of the

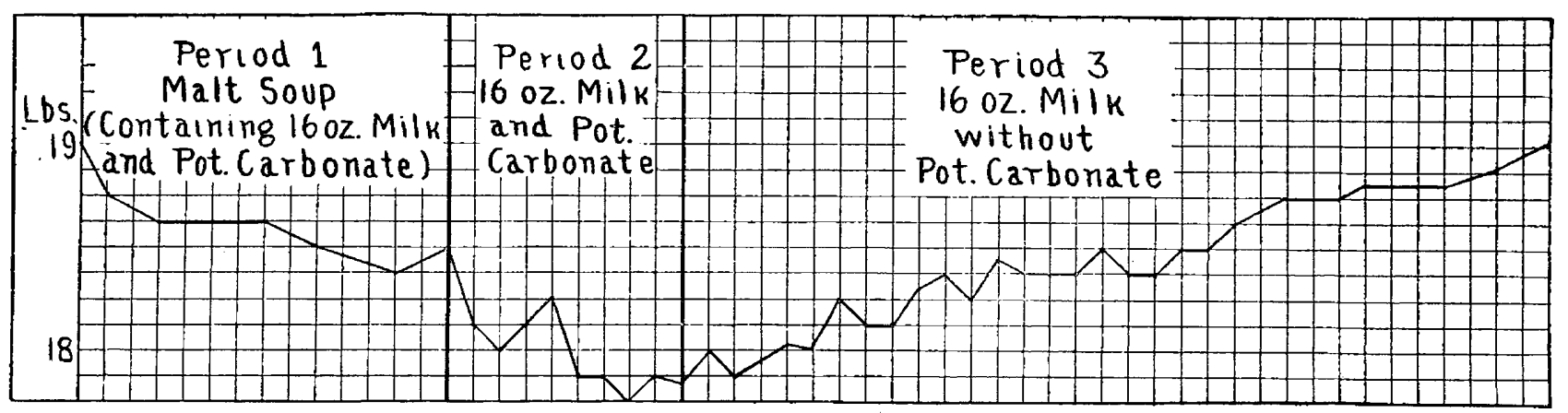

Chart 1.-Human scurvy: weight curve of a baby that developed scurvy on a diet of malt soup (Period 1). During Period 2 the flour and malt soup were omitted from the diet, the same amount of milk and potassium carbonate being continued. The disorder did not abate. The only change in Period 3 was a discontinuation of the potassium carbonate. This brought about a gain in weight and cure, showing the

sodium bicarbonate is added to a milk formula, or whether a proprietary food is more or less alkaline in reaction. To such an extent is this the case that in stating the composition of proprietary foods, the textbooks on children's diseases content themselves with a statement of the protein, fat and carbohydrate content of the various popular preparations, giving no consideration whatsoever to their reaction. In such tables the salts are expressed in terms of percentages of total ash, the nature of the inorganic constituents

4. Bohm: Boston M. \& S. J., Nov. 22, 1906, and Jan. 25, 1906.

5. Fitzwilliams, D. C. L.: Proc. Roy. Soc. Med, 2: 21, Sect. Studies on Dis. Child., 1908-1909,

6. Norbury: Proc. Roy. Soc. Med. 71. 1913

7. In addition to the references already given, the following will be found of interest:

Ridlon, J.: Report of Two Cases of Scoliosis Accompanied by Pressure Paralysis of the Lower Limbs. J. A. M. A. 67: 803 (Sept. 9) 1916.

Hodgson. F. G.: Congenital Deformities of the Vertebrae and Ribs, Am. J. Orthop. Surg. 14: 34 (Jan.) 1916

Dwight and Rotch: The Spine in Infancy, Arch. Pediat. 8:161.

Le Breton, P.: Congenital Lateral Curvature of the Spine, Pediatrics

27: 73, 1915 .

From the Bureau of Laboratories, Department of Health.

Annead before the Section on Diseases of Children at the Seventieth Annual Session of the American Medical Association, Atlantic City, water-soluble vitamin to the application of heat in an alkaline medium. There is no question, however, that this reaction renders it more susceptible to destruction. ${ }^{2}$

This subject was forced on our attention by the experience that milk formulas containing malt soup have an exceptional tendency to bring about scurvy in infants, unless an antiscorbutic foodstuff, such as orange juice or canned tomato, is added to the dietary. As has been brought out elsewhere, this undesirable effect might be the result of one or more of the following conditions: ${ }^{3}$ (1) that the formulas contain an amount of milk insufficient to protect fully; (2) that the preparation is boiled as well as frequently prepared from pasteurized milk; (3) that there is a period of aging between the initial pasteurization and the boil-

1. Hess, A. F., and Unger, L. J.: Factors Affecting Antiscorbutic

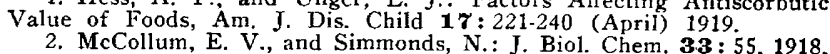
2. McCollum, E. V., and Simmonds, N.: J. Biol. Chem. 33 : 55, 1918. in water, and mixing wheat flour with milk. These two mixtures are in water, and mixing wheat flour with milk. These two mixtures are then boiled together for about three minutes. According to some direcby us, 16 ounces were employed. 
ing of the milk and the flour; (4) that an alkali is contained in the malt soup, and finally (5) that a considerable amount of carbohydrates in the form of flour and of mait sugar is added to the food. It seemed important, when an opportunity presented itself, to analyze this question, to determine which of these factors is at fault.

Chart 1 shows the weight curve of a baby that was receiving a diet of malt soup and cereal, with the addition of three teaspoonfuls of cod liver oil a day. It developed scurvy. The symptoms were those commonly associated with this disorder; namely, pallor, hemorrhages of the gums, tenderness of the femur, irritability, and lack of gain in weight. In view of this occurrence, an attempt was made to cure the scorbutic condition by eliminating the carbohydrate from the diet, by omitting the flour and malt sugar from the formula. In order to accomplish this, a pint of milk was prepared with the addition of sucrose and the same amount of potassium carbonate as is contained in the malt soup formula. ${ }^{4}$ This milk, which was fresh and of excellent quality, and contained no nalt or flour, was boiled for five minutes. There are many who believe that the carbohydrates, especially the starches, are of great importance in bringing abotit the so-called deficiency diseases, because they require a considerable amount of vitamin for their metabolism, and lead therefore to such disorders as scurvy or beriberi, if present in relatively high amount. It was found, however, that the harmful influence of the malt soup preparation could not be attributed to its high carbohydrate content, as the exclusion of the flour and the malt did not bring about an alleviation of the symptoms, or, as will be seen (Chart 1 ), lead to a gain in weight. As this form of treatment proved unsuccessful, it was determined to discontinue the addition of the potassium carbonate, so that the baby received a pint of milk that was boiled for five minutes without the addition of alkali. The sucrose, the cereal and the cod liver oil were continued. The result was striking. Not only did the infant gain in weight, as will be seen from the chart, but there was a marked change in its general condition and a disappearance of the various signs and symptoms enumerated above. It seems, therefore, quite evident that the alkalization of this food, which contains a limited amount of antiscorbutic material--merely that which is contained in a pint of boiled milk-was sufficient to render its antiscorbutic quota insufficient.

As the result of this experience, an experiment on guinea-pigs was instituted. It had been shown in previous experiments that guinea-pigs weighing about

4. This is stated as $0.44 \mathrm{gm}$, or 6.79 grains, to the fluidounce, and is measured by adding 10 c.c. of an 11 per cent. solution of potassium carbonate for each liter of prepared malt soup.
$200 \mathrm{gm}$. very rarely develop scurvy when given daily 80 c.c. per capita of fresh milk. Accordingly the same percentage of potassium carbonate as is contained in malt soup was added to this amount of milk, and its effect was noted on the weight and on the clinical condition of the guinea-pigs. Ten c.c. of an 11 per cent. solution of the alkali were mixed with raw certified milk, which was then boiled for a period of five minutes. It will be seen from Chart 2 that scurvy developed in all the pigs. That this disorder was not attributable to the alkali itself is shown by the prompt response and alleviation of symptoms, when 3 c.c. of orange juice were added to the dietary, notwithstanding the fact that the potassium carbonate was still continued (Chart 2).

These results have an application far wider than their relation merely to malt soup. Investigation brought out the fact that an alkaline potassium salt is added to the greater number of our proprietary foods for infants. This is done for one of two reasons: In the first place, because it renders the food less subject to acid fermentation, and second, because the addition of potassium counterbalances the relative poverty of this salt in cow's milk. In the majority of instances, potassium bicarbonate is added in the ratio of from 2 to 2.5 per cent., instead of the carbonate, as in malt soup in a much lower percentage. In view of the clinical and experimental results that we report, the danger of this practice is evident. In many instances it is unnecessary, as a salt such as potassium citrate probably could be substituted to supply the potassium deficiency. In cases, however, in which the object is to change the acid reaction, such a substitution would, of course, not be practicable.

\section{CONCLUSIONS}

With the realization that foods contain not only caloric factors but also accessory food factors or vitamins, it is incumbent on us to revise our standards and to reconsider their entire preparation from this new point of view. It is clear that the antiscorbutic vitamin in milk is rapidly destroyed by alkalization associated with heating, and that by the thoughtless addition of alkaline proprietary foods we are robbing the infant of much of this essential foodstuff. ${ }^{5}$ The question may be raised in this connection whether, in a similar way, we are heedlessly destroying the potency of other vitamins. It has been shown, for example, that cod liver oil is rich in at least one vitamin, the fat-soluble factor, and that it is almost a specific in

5. In a discussion published soon after infantile scurvy had come to be recognized it was asserted by a member of the Berlin Medical to be recognized, it was asserted by a member of the Berlin Medical
Society that "Riethe's albumose milk" induced scurvy. This was a vociety that "Riethe's albumose milk" induced scurvy. This was a popular preparation to which $0.4 \mathrm{gm}$. of potassium carbonate was adrict ever was paid to this observation (Meyer, E.: Berl. klin. Wchnscir. 33 : 85, 1896). 
the treatment of rickets. We have found that this oil, originally acid owing to its considerable content of organic acids, is alkaline in many of the proprietary emulsions commonly employed. It is quite possible that this alteration in reaction is not immaterial in relatian to the efficacy of this valuable therapeutic agent. 16 West Eighty-Sixth Street.

\section{ABSTRACT OF DISCUSSION}

Dr. Harry Lownenburg, Philadelphia: I should like to ask the doctor if he said that the addition of orange juice while he was administering the carbonate of potassium prevented the development of scurvy? Dr. Hess's work is very interesting and very enlightening, if it can be verified. However, it appears quite rash, on the face of it, to draw as sweeping a conclusion as he does from the study of only one sick baby and from the protocols of only four experimental guinea-pigs, in the light of vast clinical experience with many feeding cases wherein the alkalization of milk has produced no harmful effects. I have yet to see the development of scurvy in these cases. I feed many infants on malt soup extract; I never feed orange juice, although I commence the administration of comminuted green vegetables and stewed apples and prunes early. It is very difficult, indeed, to consent to have one's convictions swept aside by the meager data offered by this single case and these four experiments. I should like to see this work carried out further and confirmed. Until then I must continue to add alkali to milk mixtures where $I$ believe it to be indicated. This is said not in the light of destructive criticism. The essayist has not provided us with sufficient data. Hence hasty conclusions may not be drawn nor accepted. It is logical to conclude from the doctor's statements that those who are using malt soup extract and alkalis should have an abundance of scurvy in their practice. This has not been my experience.

Dr. Jusıus H. Hess, Chicago: When Keller first gave us the formula for making malt soup, he warned the profession against feeding this mixture for too long a period, because of the danger of developing scurvy. I have had the misfortune of placing infants on Keller's malt soup, and notwithstanding instructions the parents have remained away for from two to four months and returned with the infants showing evidences of scurvy. This has occurred three or four times. One proprietary infant's food on the market is recommended to be fed in amounts as high as three to four ounces daily. This food contains 2 per cent. of potassium bicarbonate which would result in the feeding of from 30 to 45 grains of the alkali to the quart, while the potassium bicarbonate in Keller's malt soup equals about 16 grains to the quart. A great many babies come under my observation who have been on this food for a considerable period of time, and I must confess that I have never seen a case of scurvy develop on the diet, even though it is a very one-sided one. These facts lead one to wonder why we do not see more scurvy in babies fed on the infants' foods containing potassium bicarbonate.

Dr. C. G. Kerley, New York: I have been feeding alkalis of different kinds for many years and I have had only an occasional case of scurvy, and these cases are nearly always associated with the diet of some cooked milk product and not with fresh milk. Children given fresh milk do not have scurvy whether alkalis are added or not. There must be something else, therefore, than the presence of alkalis that interferes with the vitamin content of the food. Malt soup mixtures are more apt to produce scurvy than any other diet. I never use this method of feeding without prescribing orange juice at the same time.

Dr. J. P. Crozer Griffith, Philadelphia: Let me call your attention for a moment to what is in a way ancient history, but no less important and true now, since conditions have not changed. I refer to the pulblished investigations of the American Pediatric Society some years ago, as to the effect of the feeding of different kinds of food in the production of infantile scurvy. In that publication you will find that infantile scurvy followed oftener on the use of many of the proprietary infant foods of various sorts than after any other regimen. In fact, one manufacturing company was so disturbed about the matter that they made a claim that the scurvy was due to the fact that the milk mixed with the food had been boiled. It is undoubtedly true that the boiling of milk is followed by the development of scurvy in some cases, but not nearly so often as in the use of the commercial foods. I am not referring in any way to malt soup, which is not advertised to the laity but to the profession. Dr. Hess' paper is a very valuable and interesting one. He seems to have proved in the case reported that rendering the food alkaline produced scurvy. I do not think, however, that this is convincing proof that alkalis used as a routine measure are to be feared on this account. In the American Pediatric Society's report there were mentioned ten cases of scurvy occurring in infants fed solely on breast milk. That would not prove that breast milk is to be avoided. I have fed hundreds of infants with malt soup preparations, since it is a very favorite food with me, but I have yet to see a case of scurvy following its use. It should be said, too, that in many cases the pasteurizing or sterilizing of milk serves such a very useful purpose and tends to prevent diseases so much more serious and common than scurvy, that it would be foolish to avoid its use, especially since any tendency which it may have to produce this disease can readily be obviated by the administration of orange juice. Indeed, the fact that scurvy is uncommon, considering the vast number of babies artificially fed on boiled milk, pasteurized milk, alkaline foods, and many other foods, is an indication that we do not yet know all there is to know about the etiology of this disease.

Dr. St, George T. Grinnan, Richmond, Va.: Some of you have said that scurvy is rare. It certainly is not in Richmond. I was in New York about two years ago and heard Dr. Hess read a paper and Dr. Kerley discuss it. I went back and looked up the subject of scurvy more than I had before, and $I$ have seen more cases in the last two years than $I$ had in the ten years before. I found that there was a very large sale there of a certain proprietary food, and I found many cases of scurvy in the babies fed with this food.

Dr. Joseph Grover, Boston: I feel sure that scurvy is not a rare disease. In the Children's Hospital in Boston we see one case every two, three or four days. I think boiled or pasteurized milk causes it a great deal, but the dry foods cause it more frequently still. The use of certain dry proprietary foods over a period of two or three months is fairly sure to bring on the first symptoms of scurvy.

Dr. Lewis Hill, Boston: I have used the food to which Dr. Grinnan referred a great deal and I have never seen a case of scurvy develop while it was being fed. This food is only carbohydrate with a little vegetable protein and salts. It should not be called a food. It is malt sugar and should be used as malt sugar, to which is added a reasonable amount of milk, orange juice or cereal, or some other food. I believe it is a good preparation and no more likely to produce scurvy than any other sugar is.

Dr. EfFA V. Davis, Chicago: I am rising to voice my protest against condemning a malt sugar which happens to be called a food. It is malt sugar made from wheat. We have been discussing corn syrup; dextrimaltose is now made from corn. It was formerly made from potatoes. I think we may as well face what is being placed on the market and understand it first hand, rather than condemn a thing on theory. I have tried the "malt food" in the modifying of raw milk for the past two years in my nursery, where I have from ten to fifteen artificially fed infants, without the development of scurvy. I know there is a certain percentage of potassium carbonate in the food. About six months ago, my attention was called to the fact that sodium bicarbonate added in the usual housewife's method of cooking beans destroyed the vitamins. From that hint I began to drop it in making bean soup, which I give my babies in their second year as well as the early use of antiscorbutic elements, such 
as vegetables, fruit juices, etc., from the seventh or eighth month. To go back ten years is a little ancient. We made other mistakes then which affected our results, such as feeding every two hours, for example. I think the alkalinity of infants' food is still an open question.

Dr. Emanuel C. Fleischner, San Francisco: It seems to me that we are losing sight of what Dr. Hess wishes to bring before us. I do not think it is his purpose to pass on the entire etiology of scurvy. I have seen a large number of his experimental animals, and I think no one can doulst he has demonstrated thoroughly, that with a certain fixed amount of raw cow's milk he is able to determine in an exact manner that he can keep guinea-pigs free from scurvy. Giving animals the same amount of cow's milk which under ordinary circumstances keeps them free from the condition, and by alkalizing this food to a certain extent, he has shown that the antiscorbutic vitamin is destroyed. In other words, our attention must be directed toward the fact that a high percentage of alkali in artificial foods, if we are giving a quantity of milk which is just sufficient to prevent scurvy, can destroy a sufficient amount of the vitamin in that milk to produce the clisease although it may be in a very mild state. It does not seem to me that in his paper he has tried to convey to us that all cases of scurvy are due to highly alkaline foods, and I am sure that he has not tried to prove that proprietary foods are always responsible for the disease. All of us know that we can use proprietary foods of practically all types, even with high alkaline content, if we are giving these children a large amount of raw milk. In other words, they are getting enough vitamin to prevent the development of scorbutus, notwithstanding that the alkali destroys a portion of the antiscorbutic element. It seems to me that Dr. Hess has developed a very interesting and scientific fact in this connection.

DR. F. C. NFFF, Kansas City, Mo.: I should like to ask Dr. Hess one question. Within the past year a sugar to which potassium carbonate was added was marketed with the definite statement that it was to be used instead of other preparations for the relief of constipation. I want to ask what the findings are with regard to that. I have seen no effect whatever on constipation by the addition of potassium carbonate.

Dr. A. F. Hess, New York: I do not see how we can do that, especially with the malt soup and some of the other things. Dr. Lowenburg said that he had never seen scurvy develop on malt soup. I think Dr. Kerley has answered that. If I put a bundred children on malt soup without any antiscorbutic I will get almost 100 cases of scurvy. By scurvy I do not mean the active cases that sometimes are waited for, with the bulging red gums and the hemorrhages. I mean the mild cases with pallor, loss of weight, tenderness, irritability, and a few petechiae. Those cases will develop more frequently on malt soup than on any other food. Malt soup, according to the way it is made in the final preparation, contains 0.1 per cent potassium carbonate. It is quite different from the others, and that explains why malt soup is so prone to produce scurvy. As regards the animal experiments I described only four, but I could have described many more. I do not know of any essential particular.in which animal scurvy differs from human scurvy. I have never found any. An antiscorbutic for the guinea-pig is antiscorbutic for humans. I have tested many hundreds of guineapigs in the last few years, and found that what will produce scurvy in the guinea-pig leads to it in the baby, so that we can accept, as far as I know, animal scurvy for interpretations of human scurvy. As to the dry food, I do not think that it is the drying of these proprietary foods that leads to scurvy, because, as I have recently shown, dry milk, subject to a temperature of $240 \mathrm{~F}$., for two or three seconds still retains its antiscorbutic property and when made up to the equivalent of fresh milk is able to protect guinea-pigs against scurvy.

Trained Nurse. $\rightarrow$ A trained nurse does not always enjoy being a sanitary policeman, but some one must do it if the treatment is to be carried out.-Bulletin, State Board of Health of Rhode Island, August, 1919.

\section{IMPROVISED ORTHOPEDIC EXERCISING $\triangle$ PPARATUS *}

\author{
RUDOLPH S. REICH, M.D. (Cleveland) \\ Captain, M. C., U. S. Army \\ FORT SHERIDAN, ILL.
}

During June, 1918, at Camp Cody, New Mexico, the camp orthopedic staff was confronted with the necessity of carrying out, on a large scale, many specialized corrective gymnastic exercises for soldiers and recruits among whom were found all of the whole gamut of orthopedic defects: weak feet; contractures of the finger, wrist and larger joints; limitation of motion of various joints incident to recent fractures, etc. For this work, the medical department provided the orthopedic staff absolutely nothing in the way of ready-made orthopedic apparatus, such as Zander

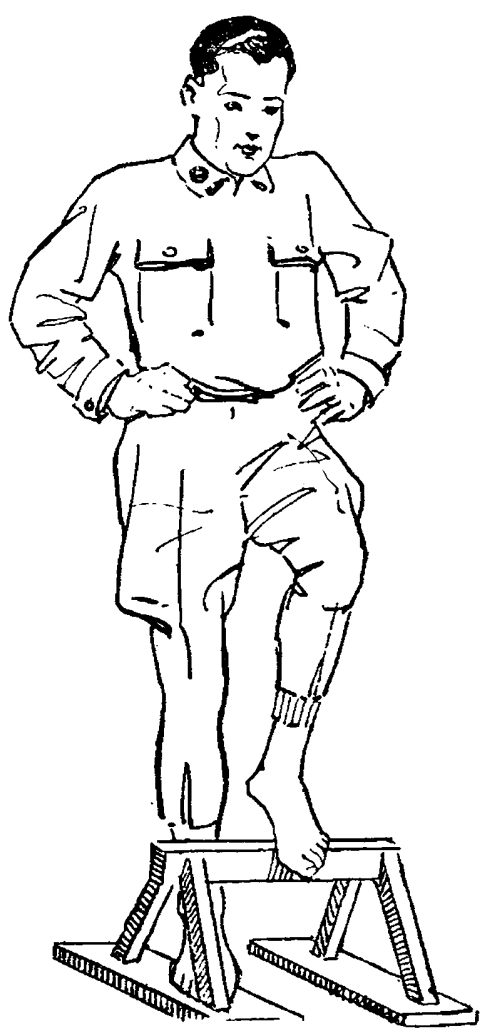

Fig. 1.-Hurdle for exercising ankle and knee. machines, and at that time the principles of vocational reconstruction had not made such appliances available. The schedule of gymnastics included specialized calisthenic exercise without apparatus, supplemented by a general daily routine suited to the individual needs of the men. Although excellent results were secured, it seemed to me that the end results might be augmented by the employment of apparatus designed to assist in corrective and developmental work.

APPARATUS FOR THE LOWER EXTREMITY

Realizing the need of apparatus to facilitate flexion and extension of the foot and knee, we devised wooden steps, having a rise of 6 inches and a tread of the same dimension, arranged in such a way that the patient walked up four and down the same number. Wooden hurdles, 8 inches high, were made (Fig. 1). These were placed at variable distances, and each man stepped over them in such a way that his foot passed directly over the hurdle, necessitating flexion and extension at the knee, and extension of the foot.

The success of this simple device, which was no doubt partly due to its novelty, encouraged us in attempting other appliances. We tried to procure an old sewing machine to stimulate dorsal and plantar flexion by pedaling, but were unable to do so, and decided to build its equivalent. Through the courtesy of the reclamation and conservation officer, we obtained a fly-wheel of a washing machine. Short pieces

* Read before the Section on Orthopedic Surgery at the Seventieth Anmual Session of the American Medical Association. Atlantic City,
N. J., June, 1919. 